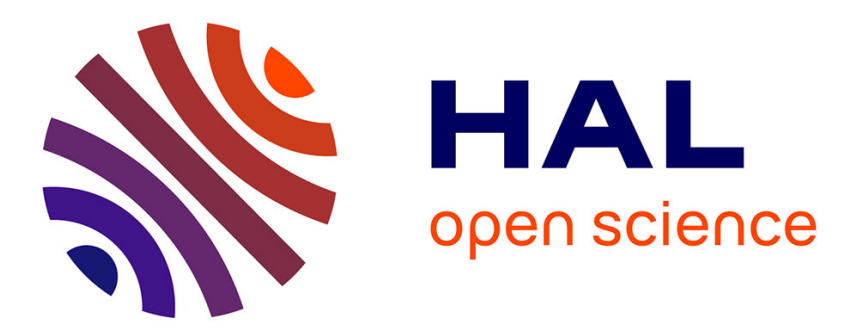

\title{
Étude d'une résonance dans une décharge en haute fréquence dans l'hydrogène en présence d'un champ magnétique statique \\ B. Grolleau
}

\section{- To cite this version:}

B. Grolleau. Étude d'une résonance dans une décharge en haute fréquence dans l'hydrogène en présence d'un champ magnétique statique. Revue de Physique Appliquée, 1974, 9 (2), pp.483-495. 10.1051/rphysap:0197400902048300 . jpa-00243807

\section{HAL Id: jpa-00243807 https://hal.science/jpa-00243807}

Submitted on 1 Jan 1974

HAL is a multi-disciplinary open access archive for the deposit and dissemination of scientific research documents, whether they are published or not. The documents may come from teaching and research institutions in France or abroad, or from public or private research centers.
L'archive ouverte pluridisciplinaire HAL, est destinée au dépôt et à la diffusion de documents scientifiques de niveau recherche, publiés ou non, émanant des établissements d'enseignement et de recherche français ou étrangers, des laboratoires publics ou privés. 


\title{
ÉTUDE D'UNE RÉSONANCE DANS UNE DÉCHARGE EN HAUTE FRÉQUENCE DANS L'HYDROGẼNE EN PRÉSENCE D'UN CHAMP MAGNÉTIQUE STATIQUE
}

\author{
B. GROLLEAU \\ Laboratoire de Physique Corpusculaire, Institut de Physique, \\ 38, boulevard Michelet, 44037 Nantes Cedex, France
}

(Reçu le 23 juillet 1973, révisé le 23 octobre 1973)

\begin{abstract}
Résumé. - Nous présentons une étude expérimentale d'un phénomène de résonance qui apparaît lorsque l'on soumet le plasma d'hydrogène d'une décharge HF, en couplage inductif, à un champ magnétique statique transversal. Nous analysons les différents paramètres qui peuvent intervenir dans le phénomène de résonance. L'expérience montre que les paramètres fondamentaux sont : la pression du gaz dans l'enceinte à décharge, l'amplitude du champ électrique induit et l'angle que fait le champ magnétique statique avec l'axe de la décharge.
\end{abstract}

Abstract. - An experimental study has been made of a resonance phenomenon which occurs in the plasma column of an inductively coupled RF discharge in hydrogen when submitted to a transverse static magnetic field. The parameters which govern this resonance phenomenon have been investigated. It is shown experimentally that the gas pressure, the amplitude of the induced electromagnetic field, and the angle between the direction of the static magnetic field and the discharge axis are the most important parameters.

1. Introduction. - La décharge en haute fréquence (en abrégé : décharge HF) ou encore décharge sans électrode est une décharge dans un gaz, entretenue par un champ électromagnétique de haute fréquence.

Ce type de décharge fut découvert par Hittorf à la fin du siècle dernier. Malgré cette ancienneté et le grand nombre d'études théoriques et expérimentales qu'elle a suscité; cette décharge n'est encore que partiellement comprise. Son étude a posé, et pose toujours, de nombreux et délicats problèmes.

Il est en effet très difficile, dans ce genre de décharge, de mettre en évidence des paramètres indépendants. Dans la plupart des systèmes, l'interdépendance des variables est telle que les études théoriques et expérimentales deviennent vite inextricables.

Dans cet article nous nous proposons d'étudier un phénomène de résonance qui apparaît dans une décharge en haute fréquence dans l'hydrogène lorsque l'on place cette décharge dans un champ magnétique statique de faible intensité. Cette étude est expérimentale, nous avons essayé de rechercher, dans la mesure du possible, les paramètres expérimentaux qui interviennent dans ce phénomène de résonance. Ce travail fait partie d'un ensemble d'études sur les décharges HF dans l'hydrogène qui ont été développées ces dernières années au Laboratoire [1], [2].

Cette résonance fut signalée pour la première fois par H. Z. Neuert [3]. De nombreux auteurs l'obser- vèrent également : J. Swingle et C. Swann [4], M. Gabovich [5], I. Ogawa et N. Abe [6], J. Williams [7]. Mais toutes ces études ont été effectuées sur des sources d'ions à excitation électromagnétique de haute fréquence, et avec pour seul objectif l'amélioration du rendement de ces sources d'ions. Pendant longtemps cette résonance n'a fait l'objet d'aucune étude pour elle-même.

Parallèlement à notre travail, un ensemble important d'études théoriques et expérimentales sur le sujet a été développé par B. Pfeiffer [8] [9], H. Oechsner [10], O. Sager [11] et plus récemment par E. P. Szuszczewicz [12]. Ces études, comme nous le verrons, traitent le problème-d'une manière expérimentale différente de celle que nous utilisons, et elles n'envisagent pas le cas d'un plasma d'hydrogène. L'interprétation théorique que présentent ces derniers auteurs n'est pas en bon accord avec nos résultats expérimentaux.

2. Dispositif expérimental. - La décharge est créée dans une enceinte cylindrique isolante. Cette enceinte est couplée inductivement à un générateur HF. Pour cela, l'enceinte à décharge est placée à l'intérieur d'une bobine excitatrice parcourue par le courant à haute fréquence. Cette bobine excitatrice est l'inductance du circuit d'accord du générateur à haute fréquence (Fig. 1). 


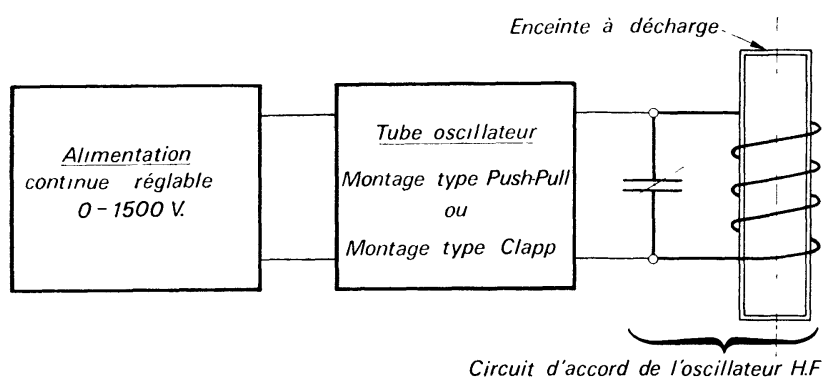

Fig. 1. - Schéma du dispositif d'excitation de la décharge.

Les générateurs HF utilisés sont constitués par des circuits auto-oscillateurs de deux types: PushPull pour les faibles puissances, inférieures à $150 \mathrm{~W}$, et Clapp pour les puissances jusqu'à $300 \mathrm{~W}$. Les caractéristiques géométriques et électriques du dispositif sont les suivantes :

Diamètre intérieur de l'enceinte à décharge en Pyrex : $34 \mathrm{~mm}$.

Longueur de l'enceinte : $180 \mathrm{~mm}$.

Diamètre moyen de l'inductance d'excitation: $50 \mathrm{~mm}$.

Nombre de spires : 4 .

Fréquence $f=\omega / 2 \pi$ de l'excitation : 20 à $50 \mathrm{MHz}$.

Stabilité de la fréquence : de l'ordre de $10^{-3}$.

Distorsion harmonique : $\simeq 1 \%$.

Puissance HF disponible : jusqu'à $300 \mathrm{~W}$.

L'enceinte à décharge est alimentée en hydrogène pur à basse pression $\left(5 \times 10^{-3}\right.$ à $10^{-1}$ torr $)$. Cette pression est contrôlée en permanence par des jauges de précision du type Pirani. D'autres gaz que l'hydrogène peuvent être admis dans l'enceinte à décharge.

A cette décharge (cf. Fig. 2), on superpose un champ magnétique statique $\mathbf{B}_{\mathrm{S}}$ dont la direction est

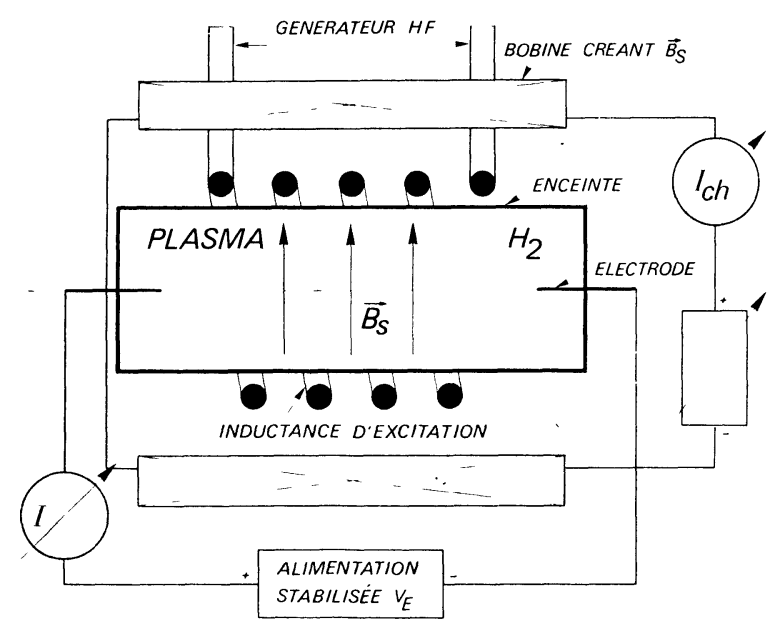

FIG. 2. - Représentation schématique du montage expérimental d'observation de la résonance.

perpendiculaire à l'axe de l'enceinte. Ce champ magnétique est de faible valeur, son intensité varie entre 0 et $70 \times 10^{-4} \mathrm{~T}$. Ce champ magnétique est créé par un groupement de bobines sans fer qui assure une uniformité convenable du champ magnétique dans tout le volume occupé par la décharge (variations relatives du champ de l'ordre de $5 \%$ ). On trouvera dans la référence [2] tous les détails concernant le dispositif expérimental.

Le dispositif d'excitation de la décharge paraît très simple dans sa conception, il entraîne cependant de sérieuses difficultés d'ordre expérimental : aux bornes de la bobine d'excitation, couplée à l'enceinte à décharge, est appliquée une différence de potentiel qui peut dépasser $1 \mathrm{kV}$ à une fréquence de quelques dizaines de mégahertz. La mesure de cette tension est délicate, les capacités d'entrées et les capacités parasites des appareils de mesure pouvant modifier sérieusement le fonctionnement du générateur HF. La mesure de la puissance HF fournie au plasma n'est pas plus aisée : en effet, l'impédance du plasma qui charge le générateur HF évolue constamment en fonction des paramètres expérimentaux de la décharge. L'indication d'un wattmètre HF n'aura donc pas beaucoup de signification. Nous nous sommes alors limités à la mesure de la puissance continue $P_{F}$ que demande le. générateur $\mathrm{HF}$ à son alimentation et nous supposons que cette puissance suit approximativement l'évolution de la puissance HF.

3. Définition et détection du phénomène de résonance. - 3.1 DÉFINITION DE LA RÉSONANCE. - En raison de la diversité des travaux sur le sujet, il est fondamental de définir nettement ce que nous appelons résonance du plasma en présence du champ magnétique statique $\mathbf{B}_{\mathrm{s}}$.

Dans notre cas, la résonance du plasma HF sous l'action du champ magnétique statique transversal $\mathbf{B}_{\mathrm{s}}$ est caractérisée par une augmentation importante de la densité électronique $n_{\mathrm{e}}$ du plasma, pour une valeur déterminée $B_{\mathrm{r}}$ du champ $B_{\mathrm{S}}$.

Dans une telle expérience, les circuits d'accord et de réglage de l'oscillateur HF restent fixes, de même que la tension continue appliquée sur l'anode du tube oscillateur. Le générateur HF délivre alors au plasma une puissance variable suivant l'adaptation plasma-générateur, c'est-à-dire en fait, suivant les caractéristiques du plasma.

Cette définition et cette méthode expérimentale sont différentes de celles adoptées par les auteurs cités dans les références [8] à [12]. Pour ces auteurs, la résonance est définie des deux manières suivantes :

- Dans les références [8] et [11], la valeur du champ $B_{\mathrm{S}}$ à la résonance, soit $B_{\mathrm{r}}$, correspond au minimum de la courbe qui traduit les variations de la différence de potentiel HF, $V_{\mathrm{HF}}$, aux bornes de la spire d'excitation, en fonction du champ magnétique statique $B_{\mathrm{S}}$. La puissance du générateur $\mathrm{HF}$ est ajustée de telle sorte que la densité du plasma $n_{\mathrm{e}}$ reste constante lorsque le champ magnétique varie. La figure 3, extraite en partie de la référence [12] donne l'allure de la courbe $V_{\mathrm{HF}}=f\left(B_{\mathrm{S}}\right)$ à $n_{\mathrm{e}}=$ Cte. 


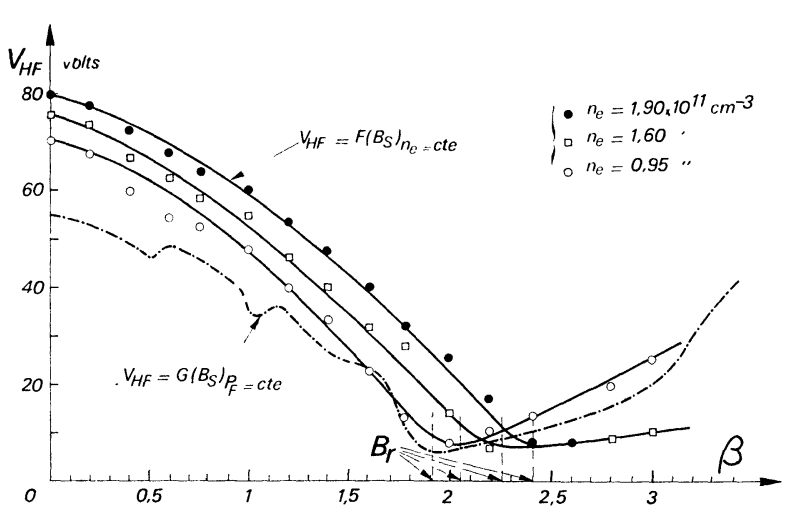

FIG. 3. - Courbes $V_{\mathrm{HF}}=f(\beta)$, avec $\beta=e B_{\mathrm{S}} / m \omega$. En traits pleins, d'après [12], en traits interrompus, d'après [10].

- Dans la référence [10], la valeur du champ $B_{\mathrm{r}}$ de résonance correspond toujours au minimum de la courbe $V_{\mathrm{HF}}$ en fonction de $B_{\mathrm{S}}$ mais, dans ce cas, on maintient constante la puissance continue $P_{\mathrm{F}}$ fournie au tube oscillateur du générateur $\mathrm{HF}$. La figure 3 donne un exemple de cette courbe $V_{\mathrm{HF}}=g\left(B_{\mathrm{S}}\right)$ à $P_{\mathrm{F}}=$ Cte.

Ces deux méthodes nécessite donc un réglage continuel de l'oscillateur HF pour chaque valeur du champ magnétique statique $B_{\mathrm{S}}$.

A ce manque d'homogénéité dans la définition de la résonance, il faut ajouter de nombreuses différences entre notre dispositif expérimental et ceux des références [8] à [12]. Ainsi dans ces travaux :

- Le circuit d'excitation est couplé inductivement au circuit d'accord du générateur HF. Ce circuit d'excitation est lui-même couplé à l'enceinte au moyen d'un ruban métallique qui entoure une fois le tube à décharge. Le point milieu de cette spire unique est relié au potentiel du sol. Ce mode d'excitation engendre une distribution du champ électromagnétique différente de celle obtenue avec notre dispositif d'excitation [13]-[14].

- Le gaz utilisé est principalement de l'argon, la pression de travail étant comprise entre $10^{-3}$ et $10^{-2}$ torr environ.

Dans nos expériences, nous utilisons surtout de l'hydrogène dans un domaine de pressions compris entre $5 \times 10^{-3}$ et $10^{-1}$ torr.

- La fréquence de travail ne dépasse jamais $27 \mathrm{MHz}$ et la plupart des résultats expérimentaux et des interprétations théoriques, se rapportent à des enceintes rectangulaires de dimensions assez grandes (70 $\mathrm{mm}$ par $160 \mathrm{~mm}$ par exemple). Lorsque des enceintes cylindriques sont utilisées, le diamètre de ces enceintes est au minimum de $60 \mathrm{~mm}$.

Dans notre cas la fréquence de travail varie entre 20 et $50 \mathrm{MHz}$ et le diamètre des différentes enceintes utilisées est compris entre 20 et $60 \mathrm{~mm}$.

Toutes ces différénces font que les comparaisons entre les résultats obtenus dans nos expériences et ceux publiés dans les références [8]-[10]-[11]-[12] s'avèrent très délicates.

3.2 DÉTECTION DE LA RÉSONANCE. - La résonance du plasma se traduisant par une augmentation de sa densité électronique $n_{\mathrm{e}}$, tous les systèmes de mesures où la densité du plasma intervient de manière significative, permettent donc d'étudier ce phénomène de résonance. Pour observer cette résonance, deux méthodes ont principalement été utilisées :

- La première méthode, consiste à suivre l'évolution du courant $I$ recueilli entre deux électrodes placées aux extrémités de l'enceinte à décharge (cf. Fig. 2). Ces électrodes sont polarisées par une tension continue stabilisée $V_{\mathrm{E}}$. La cathode étant à un potentiel très négatif par rapport à l'anode, cette électrode est à la saturation ionique tandis que l'anode est à un potentiel voisin du potentiel plasma. On démontre alors que la densité de courant $i_{\mathrm{e}}$ à la lisière de la gaine qui entoure la cathode est donnée par l'expression :

$$
i_{\mathrm{e}}=K n_{\mathrm{e}} T_{\mathrm{e}}^{1 / 2}
$$

où $T_{\mathrm{e}}$ est la température électronique, $K$ étant un coefficient qui fait intervenir la forme des électrodes et la masse des ions collectés. Si on suppose que les dimensions de la gaine évoluent peu, le courant $I$ recueilli par les électrodes est proportionnel à $i_{\mathrm{e}}$, c'est-à-dire au produit $n_{\mathrm{e}} T_{\mathrm{e}}^{1 / 2}$. Nous verrons plus loin que les variations de $T_{\mathrm{e}}$ sont petites par rapport à celles de $n_{\mathrm{e}}, I$ est donc approximativement proportionnel à la densité électronique du plasma.

- La deuxième méthode consiste à mesurer la densité électronique $n_{\mathrm{e}}$ du plasma, le long de l'axe de la décharge, au moyen d'une sonde double flottante et symétrique [15]. Les sondes sont de petites dimensions (diamètre $0,4 \mathrm{~mm}$, longueur $5 \mathrm{~mm}$ ), et les conditions expérimentales sont telles que la théorie classique des sondes doubles demeure applicable en présence du champ magnétique statique $\mathbf{B}_{\text {S }}$ [16]. Cette façon de procéder est moins souple que la précédente. Elle nécessite en effet, le relevé de la caractéristique de sonde pour chaque point de mesure, alors que la première méthode ne demande que la lecture du courant $I$. Les caractéristiques de sonde étaient enregistrées puis traitées sur un ordinateur. On obtenait ainsi la température électronique du plasma $T_{\mathrm{e}}$ et une approximation de la densité électronique $n_{\mathrm{e}}$.

- Une autre méthode intéressante pour détecter la résonance consiste à étudier les variations de la puissance continue $P_{\mathrm{F}}$ fournie par son alimentation au générateur $\mathrm{HF}$, en fonction du champ magnétique statique $\mathbf{B}_{\mathrm{S}}$. Cette puissance $P_{\mathrm{F}}$ dépend en effet de la conductivité du plasma, donc de la densité $n_{\mathrm{e}}$.

3.3 OBSERVATION EXPÉRIMENTALE DE LA RÉSONANCE. - Décrivons maintenant comment s'observe expérimentalement la résonance : 
Le tube à décharge étant rempli d'hydrogène sous une pression de 35 mtorr, on excite la décharge avec une puissance HF de quelques dizaines de watts $\left(P_{\mathrm{F}} \simeq 50 \mathrm{~W}\right.$ par exemple). Cette puissance doit être choisie suffisante pour obtenir un champ induit $\mathbf{E}_{\boldsymbol{\theta}}$ non négligeable (cf. § 4.1.1) mais cependant pas trop élevée pour éviter de se placer dans le régime inductif à forte puissance HF.

$\mathrm{Si}$ on applique progressivement le champ magnétique statique transversal $\mathbf{B}_{\mathrm{s}}$ et si l'on mesure le courant $I$ recueilli entre les deux électrodes placées aux extrémités de l'enceinte et polarisées par la tension $V_{\mathrm{E}}$, la courbe de variation $I=f\left(B_{\mathrm{S}}\right)$ est donnée par la figure 4.

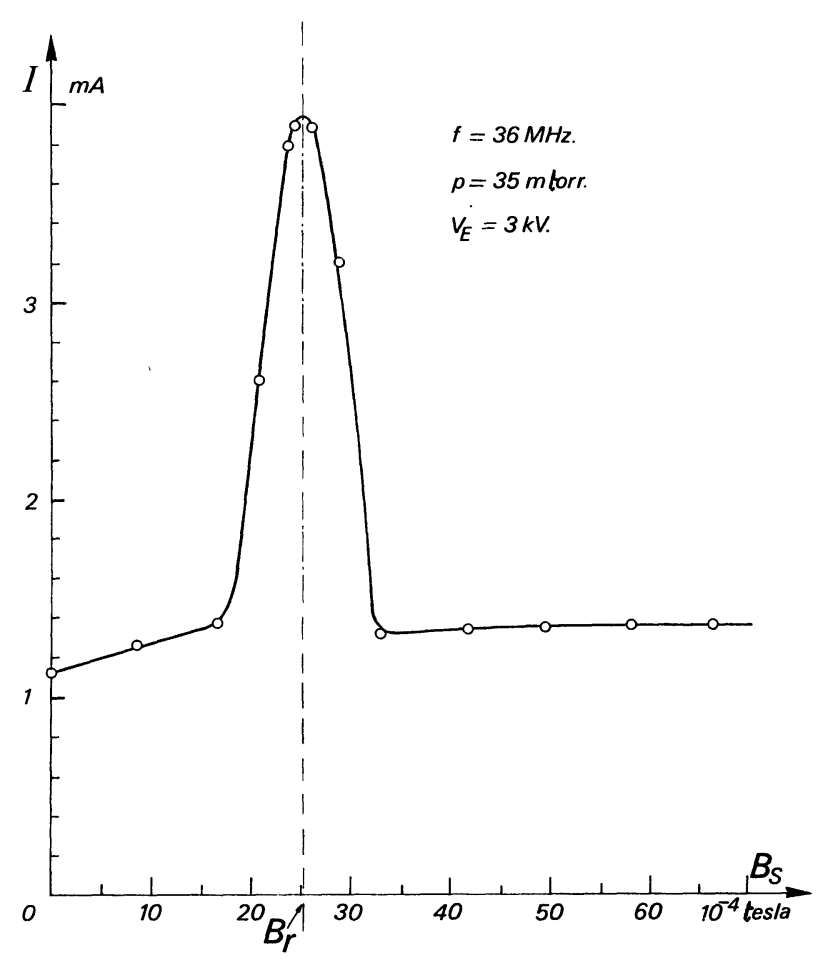

FIG. 4. - Courbe de résonance $I=f\left(B_{\mathrm{S}}\right)$. Le champ magnétique statique $B_{\mathrm{r}}$ correspondant au pic de résonance est égal à $25 \times 10^{-4}$ T environ.

Cette figure montre l'existence d'un phénomène de résonance extrêmement net pour une valeur $B_{\mathrm{S}}=B_{\mathrm{r}}$ du champ magnétique statique. Cette résonance est unique dans le domaine des champs faibles $\left(0-300 \times 10^{-4} \mathrm{~T}\right)[1]$.

Dans l'exemple de la figure 4, la fréquence d'excitation $f$ de la décharge est $36 \mathrm{MHz}$, à cette fréquence le pic de résonance apparaît pour $B_{\mathrm{S}}=B_{\mathrm{r}} \simeq 25 \times 10^{-4} \mathrm{~T}$. La fréquence gyromagnétique des électrons $f_{\mathrm{c}}$ associée à la valeur $B_{\mathrm{r}}$ du champ magnétique est :

$$
f_{\mathrm{c}}=\frac{e B_{\mathrm{r}}}{2 \pi m} \simeq 70 \mathrm{MHz}
$$

Il s'ensuit que nous avons approximativement $f_{\mathrm{c}} \simeq 2 f$. Ce résultat semble indiquer que la résonance du plasma apparaît lorsque la fréquence gyromagnétique des électrons $f_{\mathrm{c}}$ est le double de la fréquence d'excitation $f$.

4. Les différents paramètres qui contrôlent la résonance du plasma. - Pour tenter d'interpréter la résonance précédemment décrite, il est nécessaire de connaître les différentes grandeurs qui contrôlent ce phénomène. Malheureusement, dans ce type de décharge, il est souvent très difficile de trouver des paramètres indépendants les uns des autres. Le phénomène est extrêmement complexe, les interactions et les couplages entre les variables sont nombreux. Néanmoins, on peut dégager trois catégories de paramètres qui sont :

- Les paramètres liés à l'excitation de la décharge.

- Les paramètres liés au gaz de remplissage de l'enceinte.

- Les paramètres liés au champ magnétique statique $\mathbf{B}_{\mathrm{S}}$.

4.1 PARAmÈtres LIÉS A L'EXCITATION DE LA DÉCHARGE. - 4.1.1 Le type du couplage du générateur $\mathrm{HF}$ à l'enceinte à décharge. - Ce couplage détermine la distribution du champ électromagnétique à l'intérieur de la décharge. Deux types principaux de couplage sont à considérer :

- Le couplage inductif. C'est ce type de couplage que nous utilisons de manière générale. Dans ce montage, l'enceinte est à l'intérieur d'une bobine alimentée par le courant HF.

Le champ électrique possède alors deux composantes remarquables :

- Une composante $\mathbf{E}_{\theta}$, située dans le plan de section droite de l'enceinte et induite par le champ magnétique axial $\mathbf{B}_{\mathrm{HF}}$.

- Une composante longitudinale $\mathbf{E}_{\mathrm{l}}$, due au gradient de potentiel le long de la bobine d'excitation aux bornes de laquelle on applique la différence de potentiel $V_{\mathrm{HF}}$.

Si on appelle : $r$ la distance d'un point de l'enceinte à l'axe du système, $\mathbf{u}$ et $\mathbf{z}$ les vecteurs. unitaires de la tangente au cercle de rayon $r$ et de l'axe, $L$ la longueur de la bobine d'excitation, les expressions de $\mathbf{E}_{\theta}$ et de $\mathbf{E}_{1}$ s'écrivent, en première approximation et dans le vide :

$$
\mathbf{E}_{\theta}=-\frac{1}{2} r \omega B_{\mathrm{HF}} \mathbf{u} \quad \mathbf{E}_{1}=\frac{V_{\mathrm{HF}}}{L} \mathbf{z}
$$

La figure 5 donne une idée de la distribution de ces champs. N. Contaxes [17] a montré, toujours dans le vide, que les amplitudes de ces deux composantes sont reliées par la formule :

$$
\frac{E_{1}}{E_{\theta}}=\frac{2 \pi R}{p}
$$

où $R$ et $p$ sont respectivement le rayon et le pas de l'enroulement de la bobine d'excitation. Ce rapport 


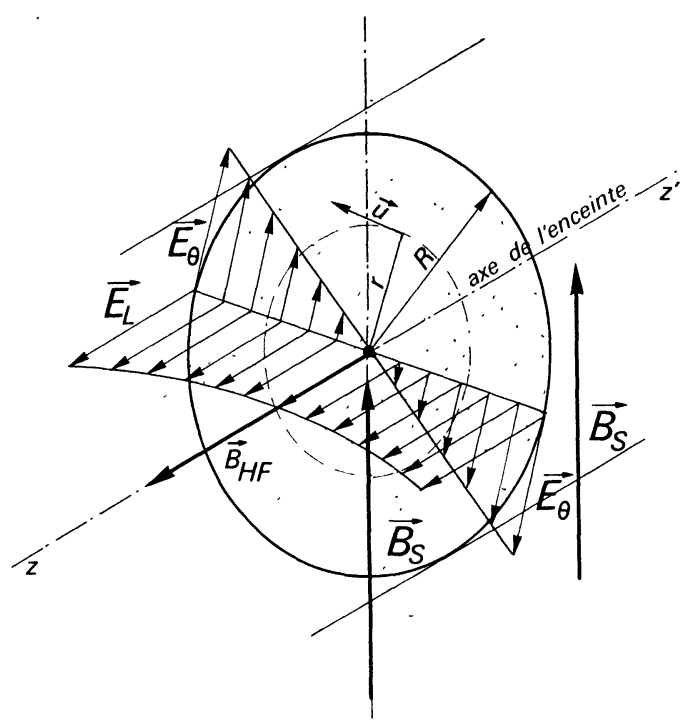

FIG. 5. - Distribution théorique du champ électromagnétique dans le vide, à l'intérieur de la bobine d'excitation.

est uniquement fonction des caractéristiques de la bobine, dans nos conditions expérimentales extrêmes il varie entre 6 et 25 . La composante $\mathbf{E}_{1}$ est ainsi toujours plus grande que $\mathbf{E}_{\theta}$ si la décharge n'est pas blindée. $E_{\theta}$ est au plus de l'ordre de 10 à $15 \mathrm{~V} / \mathrm{cm}$, la composante longitudinale ne dépasse pas 60 à $80 \mathrm{~V} / \mathrm{cm}$. Nous verrons dans la suite que diverses méthodes permettent d'agir séparément sur ces deux composantes.

La distribution de la figure 5 doit être modifiée lorsque le plasma est présent dans l'enceinte [18]-[19]. Cependant en l'absence de champ magnétique statique, nos conditions expérimentales sont telles que cette distribution n'est pas trop modifiée [2].

- Le couplage capacitif. Dans ce tube de couplage, l'enceinte à décharge est située entre les deux armatures d'un condensateur plan aux bornes duquel on applique la tension HF. Le champ électrique $\mathbf{E}$ est alors parallèle à l'axe de l'enceinte à décharge et est sensiblement uniforme à l'intérieur du plasma. Ce type de couplage engendre donc une distribution du champ électromagnétique plus simple que dans le cas du couplage inductif.

4.1.2 La fréquence $f$ d'excitation de la décharge. Le dispositif expérimental permet de faire varier la fréquence $f$ entre 20 et $50 \mathrm{MHz}$. Dans ce domaine, nous pourrons suivre l'influence de la fréquence sur la valeur $B_{\mathrm{r}}$ du champ magnétique de résonance.

4.1.3 Le niveau de la puissance HF disponible au générateur d'excitation. - Le générateur HF fournit l'énergie électromagnétique au plasma mais la puissance demandée par le plasma dépend des caractéristiques de ce plasma et réciproquement. Lorsque le champ magnétique statique $\mathbf{B}_{\mathrm{S}}$ varie, la puissance fournie au plasma varie, elle passe par un maximum à la résonance. On peut choisir le niveau de la puissance à $B_{\mathrm{S}}=0$ et à la résonance en fixant la valeur de la tension plaque $V_{\mathrm{p}}$ du tube oscillateur du générateur HF.

4.2 PARAmÈtres liés AU GAZ DE REMPlisSAGE DE L'ENCEINTE. - 4.2.1 La pression $p$ du gaz. - Ce paramètre est fondamental dans nos expériences, la résonance n'existant qu'à l'intérieur d'un intervalle étroit de pressions. La pression détermine la fréquence des collisions électron-neutre $v_{\mathrm{e} 0}$. Ainsi dans l'hydrogène et si l'énergie des électrons est supérieure à $4 \mathrm{eV}$, on a d'après W. P. Allis [20] : $v_{\mathrm{e} 0}=1,59 \times 10^{9} \mathrm{p}$. La pression interviendra donc dans l'expression de la conductivité $\sigma$ du plasma, on a en effet :

$$
\sigma=G\left(n_{0}, r, v_{\mathrm{e} 0}, \omega, B_{S}\right)
$$

( $n_{0}$ densité électronique sur l'axe), et cette conductivité joue un rôle important dans le couplage générateurplasma.

4.2.2 La nature du gaz. - Il est également intéressant d'étudier l'influence de la nature du gaz sur le phénomène de résonance. La fréquence de collision $v_{\mathrm{e} 0}$ est différente d'un gaz à l'autre, ce fait peut modifier le comportement de la résonance en fonction de la pression. D'autre part, l'étude de la résonance avec des gaz différents permet de voir dans quelle mesure la nature des ions du plasma intervient. Nous avons utilisé successivement l'hydrogène, l'air et l'argon. Il faut cependant noter que les études les plus complètes ont été effectuées avec l'hydrogène.

4.3 PARAMÈTReS LIÉS AU CHAMP MAGNÉTIQUE STATIQUE $\mathbf{B}_{\mathrm{S}}$. - Nous pouvons agir sur son amplitude et sur sa direction. La variation de l'amplitude est continue puisque ce champ $\mathbf{B}_{\mathbf{S}}$ est créé par des bobines sans fer. La direction du champ magnétique statique est soit perpendiculaire à l'axe de l'enceinte, soit parallèle, soit inclinée d'un angle $\psi$ sur l'axe. Dans ce dernier cas, le champ $\mathbf{B}_{\mathrm{S}}$ est obtenu par un groupement de bobinages.

5. Résultats expérimentaux. Influence des divers paramètres sur la résonance. - Après avoir recensé les principaux paramètres expérimentaux qui peuvent intervenir, examinons leur influence respective sur le phénomène de résonance décrit au paragraphe 4.2.

5.1 INFLUENCE SUR LA RÉSONANCE DU MODE DE COUPlAGE PlASMA-GÉNÉRATEUR. - La courbe de la figure 4 montre que, si le couplage du générateur et du plasma est inductif, la résonance existe pour des conditions particulières de pression et d'excitation. Si l'on reprend ces mêmes conditions de pression et d'excitation mais avec un couplage capacitif entre le générateur $\mathrm{HF}$ et le plasma, aucun phénomène de résonance n'apparaît. Sur la figure 6 on constate que le champ magnétique statique $\mathbf{B}_{\mathrm{S}}$ n'a pratiquement aucune action sur la décharge. La modification des conditions expérimentales (pression, puissance $\mathrm{HF}$, champ magnétique) ne change pas ce résultat.

Les mêmes expériences ont été reprises avec non plus le champ $\mathbf{B}_{\mathrm{S}}$ transversal mais avec $\mathbf{B}_{\mathrm{S}}$ axial 


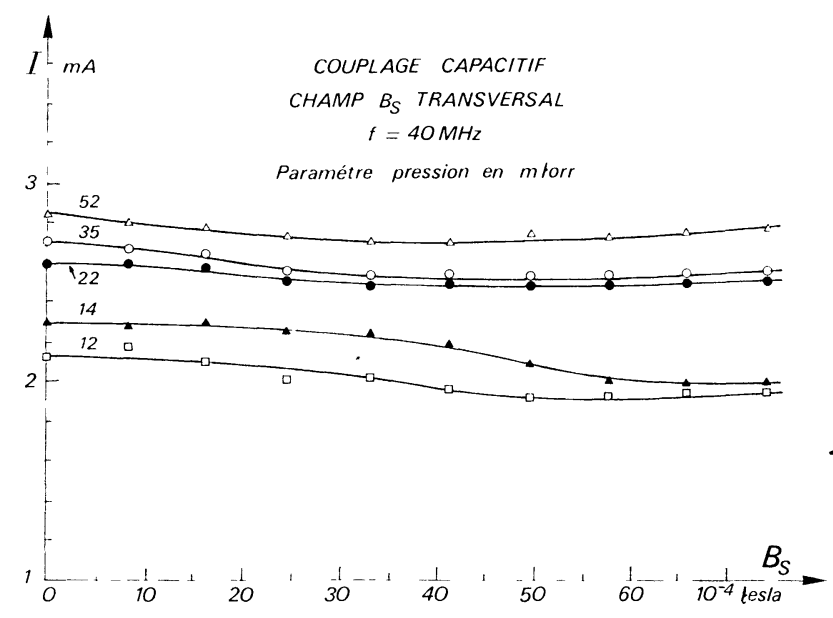

Fig. 6. - Variation du courant $I$ en fonction du champ magnétique statique. Le couplage entre le générateur et le plasma est du type capacitif.

c'est-à-dire parallèle à l'axe de l'enceinte. La figure 7 indique que le champ $\mathbf{B}_{\mathrm{S}}$ entraîne une légère augmentation de la densité du plasma mais résonance aucune n'apparaît.

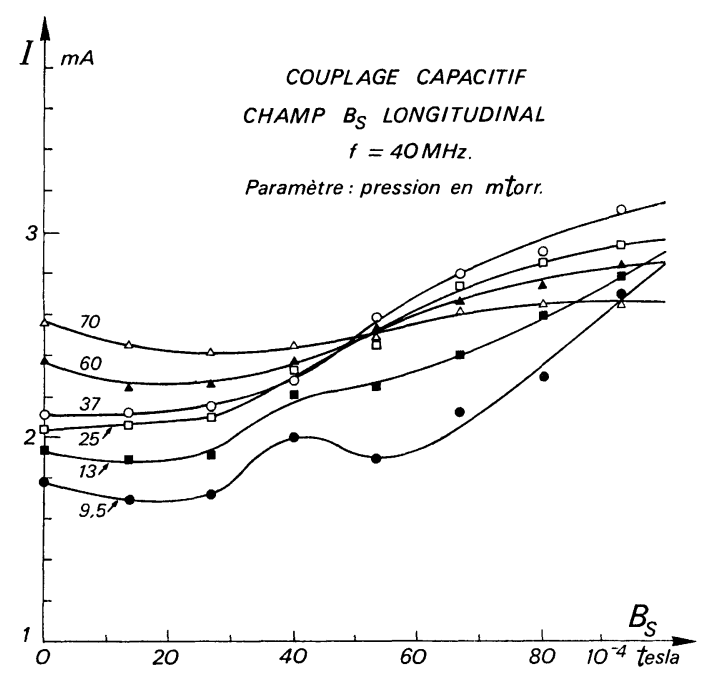

FIg. 7. - Action du champ magnétique statique longitudinal $\mathbf{B}_{\mathrm{S}}$, en couplage capacitif.

D'après ces résultats, il semble que la résonance que nous obșervons soit liée à l'existence du champ électrique induit $\mathbf{E}_{\theta}$, ce champ n'étant présent que lors d'un couplage inductif entre la décharge et le générateur.

5.2 INFLUENCE DE LA FRÉQUENCE D'EXCITATION. Nous avons vérifié que la relation $f_{\mathrm{c}} \simeq 2 f$ était bien satisfaite dans toute la gamme des fréquences que pouvaient délivrer nos générateurs HF. La bobine d'excitation utilisée dans ces expériences était toujours la même, de cette façon on est assuré que la distribution des champs HF n'est pas modifiée au niveau de l'excitation, nous verrons en effet que cette distribution influe sur la forme de la courbe de résonance.
La figure 8 permet de vérifier que la relation linéaire $B_{\mathrm{r}}=\eta f$ entre le champ statique à la résonance $B_{\mathrm{r}}$ et la fréquence d'excitation $f$ est satisfaite. Dans

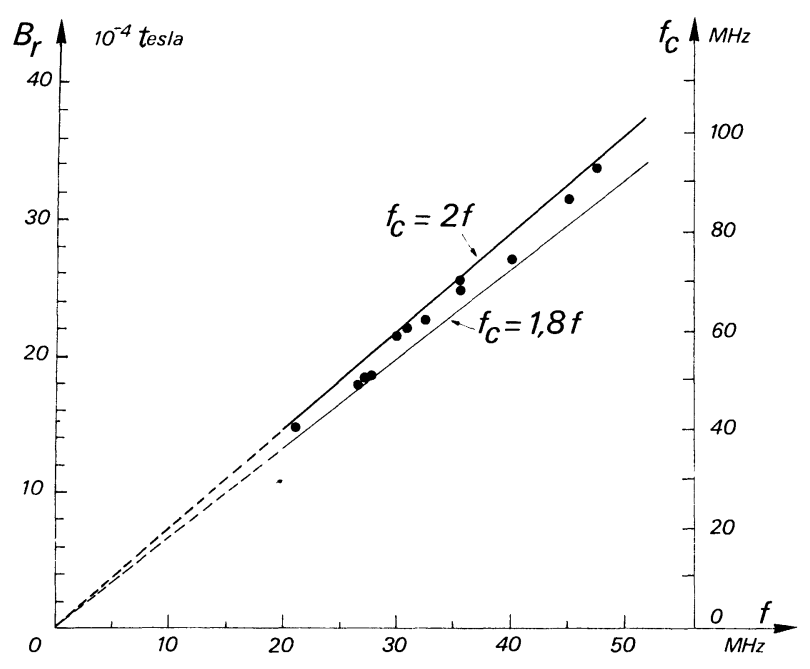

Fig. 8. - Vérification de la dépendance linéaire entre le champ de résonance $B_{\mathrm{r}}$ et la fréquence d'excitation $f$.

l'ensemble des expériences, on constate que la fréquence gyromagnétique $f_{\mathrm{c}}$ est toujours très légèrement inférieure à $2 f$ soit :

$$
1,8 f<f_{\mathrm{c}}<2 f .
$$

Cet écart ne peut pas être attribué aux erreurs expérimentales. Nous n'avons pas actuellement d'interprétation théorique permettant de justifier la relation $f_{\mathrm{c}} \simeq 2 f$.

5.3 INFLUENCE DE LA NATURE DU GAZ. - Lorsque nous avons relié la valeur du champ magnétique de résonance $B_{\mathrm{r}}$ à la fréquence gyromagnétique $f_{\mathrm{c}}$, nous avons supposé implicitement que les particules intéressées étaient les électrons. On admet ainsi que le mouvement des ions est peu influencé par les champs HF, en raison de leurs masses élevées.

Pour vérifier que la nature du gaz, par l'intermédiaire de la masse des ions et des fréquences de collisions $v_{\mathrm{e} 0}$, n'a pas d'influence sur l'existence de la résonance, nous avons repris les expériences avec l'air et l'argon. Dans des conditions expérimentales presque identiques aux précédentes, la résonance apparaît dans ces deux gaz pour $f_{\mathrm{c}} \simeq 2 f$. Cependant, dans l'argon, le phénomène de résonance est beaucoup moins important que dans le cas de l'hydrogène. I. Ogawa [6] a également obtenu une résonance pour $f_{\mathrm{c}} \simeq 2 f$ dans l'hélium.

En conclusion des résultats des expériences faisant intervenir la fréquence d'excitation $f$ et la nature du gaz, il semble logique de penser que ce sont les électrons du plasma qui sont à l'origine du phénomène de résonance.

5.4 INFLUENCE DE LA PRESSION DU GAZ DANS L'ENCEINTE SUR L'EXISTENCE DE LA RÉSONANCE. - La réSO- 
nance est étroitement liée à la pression du gaz dans l'enceinte à décharge. Elle n'existe que dans un intervalle déterminé de pressions.

Pour l'hydrogène, la limite inférieure de cet intervalle, soit 10 mtorr, est indépendante de la fréquence d'excitation $f$ et de la puissance HF fournie à la décharge. Par contre, la limite supérieure qui varie entre 50 et 70 mtorr semble liée à la puissance $\mathrm{HF}$. Plus cette puissance est élevée et plus la limite se déplace vers les hautes pressions. Ainsi il était impossible d'obtenir une résonance à 70 mtorr avec un générateur $\mathrm{HF}$ de faible puissance $\left(P_{\mathrm{F}} \simeq 100 \mathrm{~W}\right)$ alors que celle-ci apparaît faiblement avec un générateur'délivrant une puissance de l'ordre de $300 \mathrm{~W}$.

Pour une puissance $\mathrm{HF}$ déterminée (à champ $B_{\mathrm{S}}$ nul), la résonance n'apparaît que dans un domaine de pressions bien défini que nous avons précisé [21]. L'amplitude de la résonance est cependant variable à l'intérieur de ce domaine. Entre 15 et 30 mtorr, l'amplitude de la résonance est maximum, elle décroît de part et d'autre de ces valeurs.

L'existence de ce domaine de pressions se voit clairement sur la figure 9.

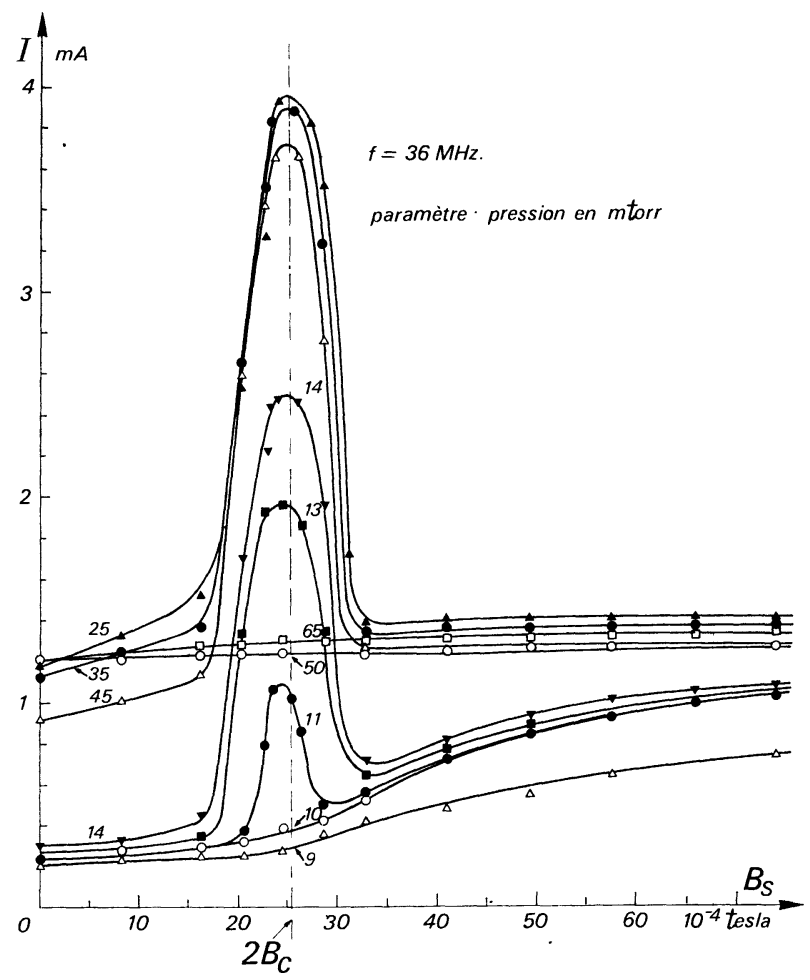

Fig. 9. - Evolution de la résonance en fonction de la pression de l'hydrogène dans l'enceinte à décharge.

5.5 RÔLE DE L'AMPLITUDE DES CHAMPS ÉLECTRIQUES HF OU DU NIVEAU DE LA PUISSANCE HF DISPONIBLE AU GÉNÉRATEUR. - La pression n'est pas le seul paramètre qui intervient pour déterminer l'apparition de la résonance. L'amplitude du champ électromagnétique est également un facteur prépondérant.

Lorsque, en dehors de la résonance, la puissance HF fournie au plasma est petite (dans nos expériences cela signifie en général que la tension plaque $V_{\mathrm{p}}$ appliquée sur le tube oscillateur est faible), on n'obtient pas de résonance. Il est nécessaire que le champ électrique induit $\mathbf{E}_{\theta}$ ait au départ une certaine amplitude, c'est-à-dire que la puissance HF soit suffisamment élevée, pour que la résonance apparaisse.

$\mathrm{Si}$, au contraire, la puissance communiquée à la décharge est' très importante (de l'ordre de $200 \mathrm{~W}$ au minimum dans nos conditions expérimentales), la décharge est dans le régime brillant ou induit (cela suppose que la pression est supérieure à 20 mtorr dans l'hydrogène); le champ magnétique $\mathbf{B}_{\mathrm{S}}$ n'a alors plus d'influence notable sur le plasma. Tout se passe comme si le plasma avait atteint un régime optimum.

Entre ces deux extrêmes, l'influence de l'amplitude du champ électromagnétique HF apparaît dans l'évolution de la forme de la courbe de résonance $I=f\left(B_{\mathrm{S}}\right)$. La figure 10 montre cette évolution lorsque la puissance $\mathrm{HF}$ fournie à la décharge augmente.

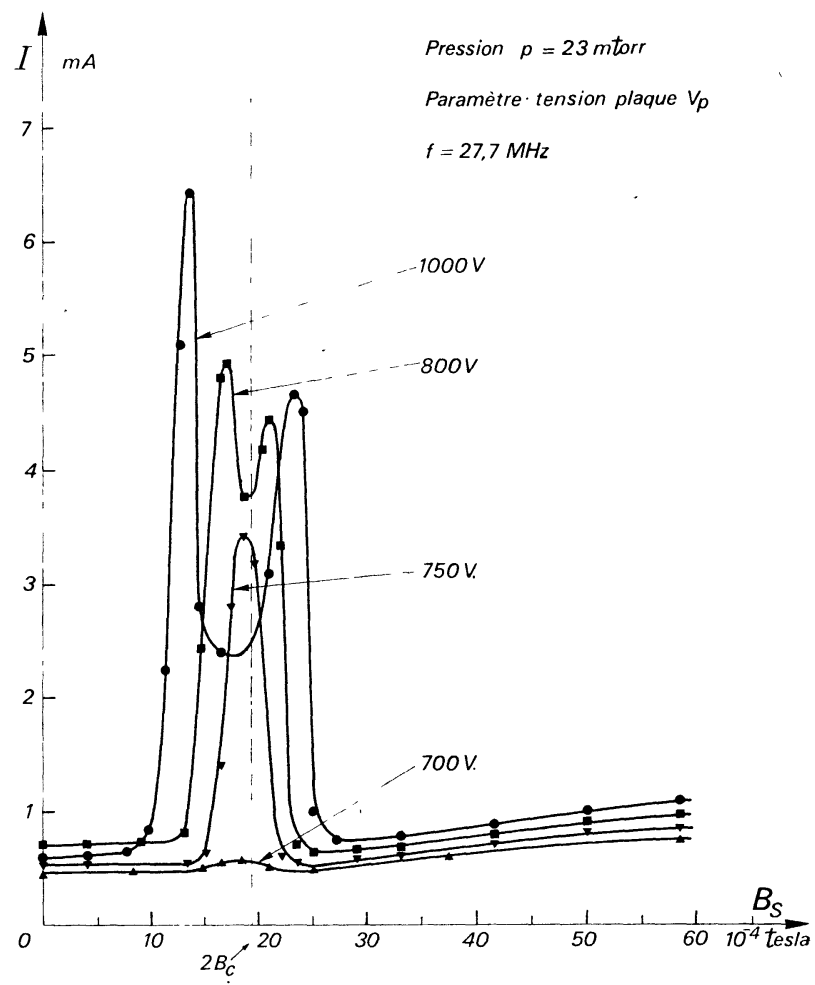

Fig. 10. - Evolution de la courbe de résonance en fonction de la puissance HF disponible au générateur.

On remarque sur cette figure deux modifications par rapport à la figure 3 :

- L'élargissement de la courbe de résonance lorsque la puissance $\mathrm{HF}$ fournie à la décharge augmente. Dans le paragraphe 5.7.3 nous montrons que cet élargissement est vraisemblablement dû à l'accroissement de la composante longitudinale du champ avec la puissance.

- L'apparition de deux maxima sur la courbe de résonance. Si le générateur $\mathrm{HF}$ est peu puissant 
$\left(P_{\mathrm{F}}<100 \mathrm{~W}\right)$ la courbe de résonance ne présente qu'un seul maximum centré sur le champ magnétique $B_{\mathrm{S}}=B_{\mathrm{r}}$, tel que $f_{\mathrm{c}} \simeq 2 f$. Lorsque l'on augmente la puissance HF fournie à la décharge, deux pics apparaissent, ces pics se placent sur deux valeurs de $B_{\mathrm{S}}$ sensiblement symétriques par rapport à $B_{\mathrm{r}}$. L'intervalle entre ces deux pics croît en même temps que la puissance HF. L'existence de ces deux maxima n'est pas clairement comprise. Une interprétation possible serait que dans ce cas, la charge de l'oscillateur devient trop importante et que des modifications dans le couplage et dans l'adaptation plasma-générateur $\mathrm{HF}$ interviennent. Il faut également signaler que pour une valeur du champ magnétique statique $B_{\mathrm{S}}$, correspondant au creux entre les deux maxima, le signal HF capté par une spire entourant l'enceinte à décharge, contient des signaux à des fréquences de l'ordre de 200 à $300 \mathrm{kHz}$, suivant les conditions d'excitation. Ces signaux n'existent pas lorsque la courbe de résonance ne possède qu'un maximum.

5.6 INFLUENCE DE LA PRESSION SUR LA FORME DE LA COURBE DE RÉSONANCE. - L'amplitude du champ électromagnétique dans le plasma n'est pas uniquement commandée par les conditions de fonctionnement du générateur HF (tension ou puissance appliquée sur la plaque du tube oscillateur par exemple), elle dépend, par l'intermédiaire de la conductivité $\sigma$ du plasma, de la pression du gaz dans l'enceinte à décharge.
A puissance HF constante en l'absence de champ magnétique statique, la densité du plasma varie assez peu en fonction de la pression. Il n'en est pas de même pour la forme de la courbe de résonance qui évolue en fonction de la pression.

La figure 11 montre les modifications de la courbe de résonance lorsque la pression $p$ varie à l'intérieur de l'intervalle 10-70 mtorr. La puissance $\mathrm{HF} P_{\mathrm{F}}$, était maintenue à une valeur constante pour $B_{\mathrm{S}}=0$.

Pour les pressions situées vers la limite inférieure de l'intervalle d'existence de la résonance, la courbe possède un pic de résonance centré sur $B_{\mathrm{S}}=B_{\mathrm{r}}$, il en est de même pour les pressions situées vers la limite supérieure de l'intervalle. Entre ces valeurs la courbe de résonance présente deux maxima situés de part et d'autre de $B_{\mathrm{r}}$, l'écartement des pics et leur amplitude étant maxima pour une pression voisine de 20 mtorr.

\subsection{RÔLES RESPECTIFS DES DIFFÉRENTES COMPOSANTES} DU CHAMP ÉLECTRIQUE HF. - Nous nous proposons maintenant d'examiner quelle est l'influence respective du champ induit $\mathbf{E}_{\theta}$ et du champ longitudinal $\mathbf{E}_{1}$ sur l'existence et sur l'évolution de la résonance. Pour cette étude, nous avons utilisé différents moyens qui permettent de faire varier ces champs, principalement :

- Un écran placé entre la bobine d'excitation et le tube à décharge. Il est constitué par un cylindre

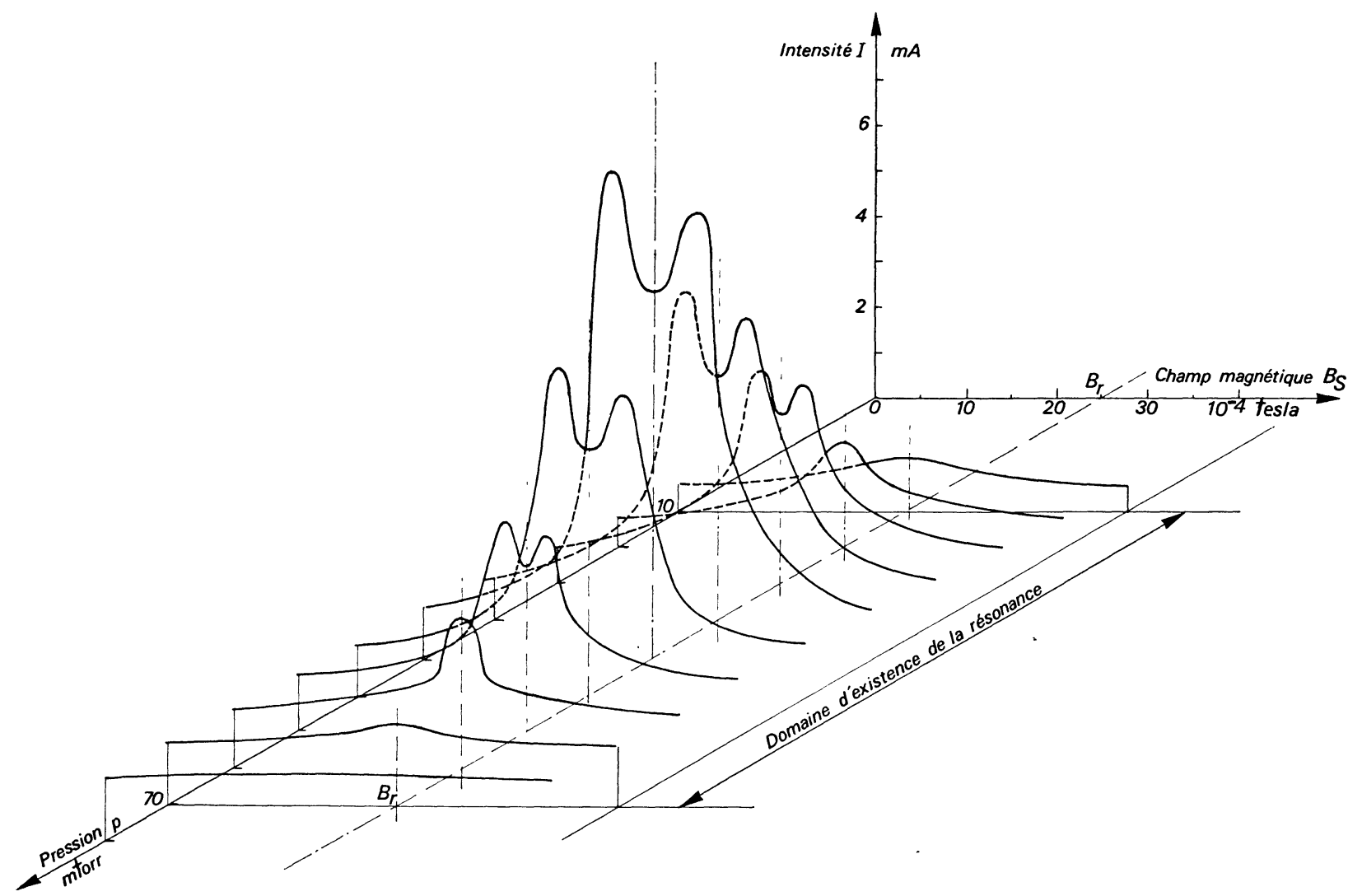

FIG. 11. - Evolution de la forme de la courbe de résonance à l'intérieur de l'intervalle de pressions (à $B \mathrm{~S}=0, P_{F}=$ Cte). 


\section{TABLEAU I}

Caractéristiques électriques des bobines d'excitation de la décharge

$\begin{array}{ccccc}\text { Pas }(\mathrm{mm}) & \begin{array}{c}\text { Facteur } \\ \text { de surtension } \mathrm{Q} \\ (20 \mathrm{MHz})\end{array} & \begin{array}{c}\text { Inductance } \\ L(\mu \mathrm{H})\end{array} & \begin{array}{c}\text { Fréquence } \\ \text { du générateur } \\ (\mathrm{MHz})\end{array} & \begin{array}{c}E_{1} / E_{\theta} \\ (\mathbf{a} r=R) \\ 11\end{array} \\ - & 460 & - & - & - \\ 12,5 & 450 & 0,712 & 29,9 & 13,2 \\ 15 & 424 & 0,682 & 30,9 & 11,6 \\ 17,5 & 414 & 0,651 & 31 & 9,7 \\ 20 & 410 & 0,597 & 31,25 & 8,3 \\ & & 0,577 & 32 & 7,3\end{array}$

de cuivre de $1 \mathrm{~mm}$ d'épaisseur dans lequel on a taillé sur huit génératrices des rainures de $4 \mathrm{~mm}$ de large. Seule une des rainures débouche aux deux extrémités du cylindre, on empêche ainsi un trop fort couplage entre l'écran et le générateur $\mathrm{HF}$ en évitant de constituer des circuits fermés. L'écran est beaucoup plus long que la bobine d'excitation (2,5 fois sa longueur). N. Dodge [22] a mesuré, dans le vide, l'efficacité d'un écran de ce type. Le champ longitudinal $\mathbf{E}_{1}$ est réduit de $75 \%$ lorsque l'écran est présent.

- Une bobine d'excitation construite de manière que le courant circule en sens inverse dans deux spires adjacentes. Le champ induit $\mathbf{E}_{\boldsymbol{\theta}}$ doit être globalement nul.

- Des bobines d'excitation avec des pas d'enroulement différents mais qui possèdent le même nombre de spires. De cette façon on peut faire varier, comme nous l'avons vu au paragraphe 4.1 .1 , le rapport $E_{1} / E_{\theta}$ en faisant varier $E_{1}$. Il suffit pour cela de maintenir constante l'amplitude de la tension HF appliquée aux bornes des bobines. Le tableau I résume les caractéristiques de ces bobines.

Ce tableau montre que les caractéristiques électriques des bobines d'excitation varient peu. Le fonctionnement à vide du générateur $\mathrm{HF}$, sera ainsi identique pour toutes les bobines.

5.7.1 Résonance dans le cas de la décharge blindée. Lorsque l'écran est en place il est impossible d'amorcer la décharge. Cela s'explique par le fait que le champ $\mathbf{E}_{\boldsymbol{\theta}}$ a une amplitude insuffisante pour engendrer l'ionisation du gaz. Si la décharge a été préalablement amorçée, l'introduction de l'écran ne supprime que la zone de décharge qui est excitée capacitivement. La partie induite de la décharge n'est pas perturbée, le champ d'entretien pouvant être très faible. L'expérimentation avec une décharge blindée exige donc que la puissance HF fournie à la décharge crée un champ induit $\mathbf{E}_{\theta}$ suffisant pour qu'apparaisse le régime induit.

La figure 12 montre l'évolution de la courbe de résonance en présence de l'écran. Cette courbe a même allure que celle obtenue sans le blindage, cependant la largeur à mi-hauteur a sensiblement diminué (sur cette figure, le rapport des largeurs est de l'ordre de 0,7 ).

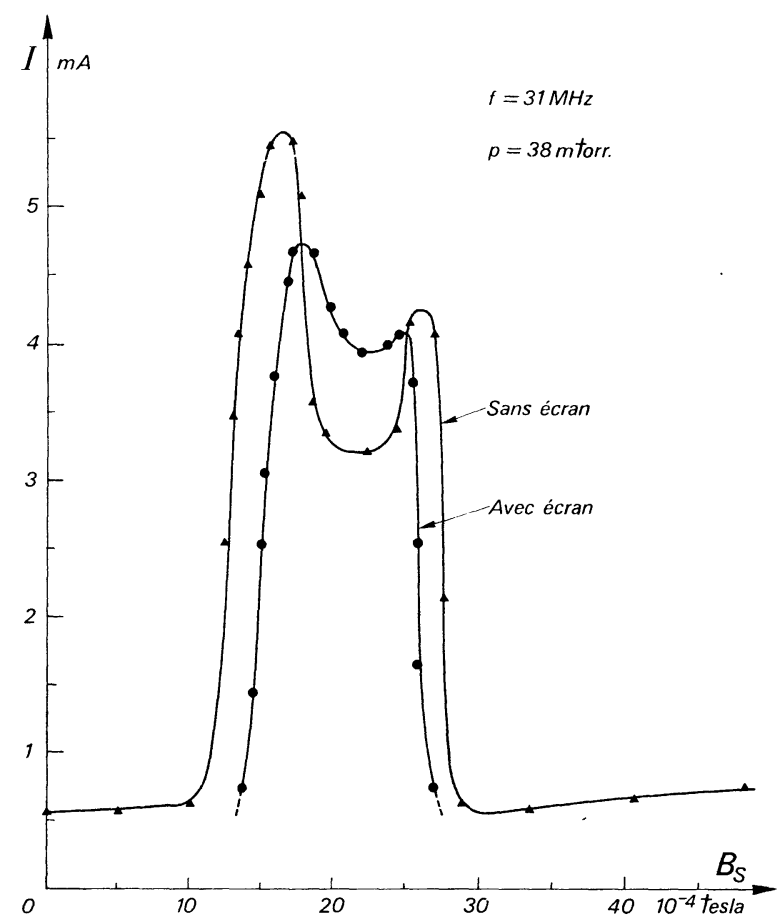

FIG. 12. - Courbe de résonance avec et sans blindage du champ longitudinal $\mathbf{E}_{1}$

Ce résultat semble prouver d'une part que c'est le champ induit $\mathbf{E}_{\theta}$ qui est responsable de la résonance et d'autre part que le champ longitudinal $\mathbf{E}_{1}$ intervient pour déterminer la largeur de la courbe de résonance.

5.7.2 Résonance au moyen d'une bobine sans champ induit $\mathbf{E}_{\boldsymbol{\theta}}$. - Dans ce cas il n'est pas possible d'obtenir une résonance du plasma. Le champ magnétique $\mathbf{B}_{\mathrm{S}}$ est sans action sur la décharge. Ce résultat confirme l'hypothèse que la présence du champ induit $\mathbf{E}_{\theta}$, avec une amplitude suffisante, est nécessaire pour obtenir la résonance.

5.7.3 Rôle de l'amplitude du champ longitudinal $\mathbf{E}_{\mathbf{1}}$ sur la largeur de la courbe de résonance. - En maintenant constante la tension $V_{\mathrm{HF}}$ aux bornes des bobines d'excitation ( $V_{\mathrm{HF}} \simeq 1500 \mathrm{~V}$ crête à crête), la diminution du pas doit entraîner un accroissement $\mathrm{du}$ champ longitudinal $\mathbf{E}_{\mathbf{l}}$. 
L'expérimentation montre que les courbes de résonance ont l'allure classique, cependant la largeur à mi-hauteur de la courbe est d'autant plus faible que le pas est grand c'est-à-dire que le champ électrique longitudinal est faible.

La figure 13 montre comment évolue la largeur relative à mi-hauteur $\Delta B_{\mathrm{S}} / B_{\mathrm{r}}$ lorsque le pas $p$ varie. $\mathrm{Ce}$ résultat montre bien que la largeur de la courbe de résonance est liée à l'amplitude du champ électrique longitudinal $\mathbf{E}_{1}$.

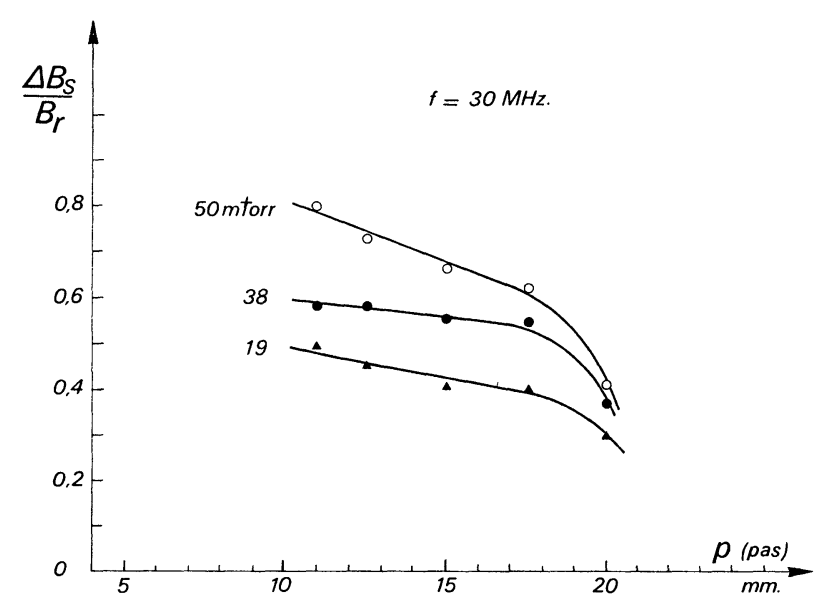

FIG. 13. - Evolution de la largeur relative à mi-hauteur de la courbe de résonance lorsque le pas de la bobine d'excitation. varie.

Remarque. - Il est important de noter que, dans toutes ces expériences, nous avons considéré seulement les variations (sans d'ailleurs les calculer) du champ $\mathbf{E}_{1}$ dans le vide.

La mesure de $\mathbf{E}_{1}$ à l'intérieur du plasma est difficile dans le cas d'une décharge en haute fréquence en couplage inductif. On peut cependant supposer que le champ $\mathbf{E}_{1}$ créé par la bobine d'excitation est affaibli à l'intérieur du plasma, d'une part du fait de l'existence d'une gaine de charge d'espace à la transition plasma-enceinte et d'autre part par un effet de pénétration dû à la conductivité du plasma.

5.8 INFLUENCE DE LA DIRECTION DU CHAMP MAGNÉTIQUE STATIQUe $\mathbf{B}_{\mathrm{S}}$. - Nous avons étudié l'évolution $\mathrm{du}$ phénomène de résonance lorsque l'on fait varier la direction du champ magnétique statique $\mathbf{B}_{\mathrm{S}}$ par rapport à l'axe de l'enceinte.

Pour cela, les expériences en couplage inductif ont été reprises en utilisant un champ magnétique $\boldsymbol{B}_{\boldsymbol{S}}$ axial. Ce champ était produit par des bobines de Helmholtz, il était bien homogène à l'intérieur du volume de la décharge.

La figure 14 donne le résultat de ces expériences. On remarque sur cette figure que le champ magnétique $\boldsymbol{B}_{\mathrm{S}}$ n'entraîne qu'une petite augmentation de la densité du plasma sans toutefois présenter un caractère de résonance, et ceci quelles que soient la pression et les conditions d'excitation de la décharge. Ce résultat

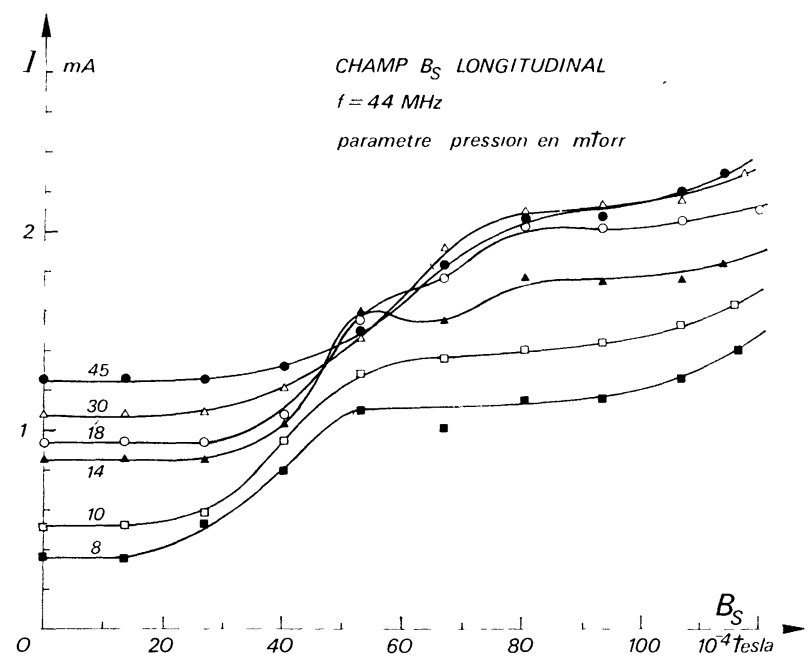

Fig. 14. - Etude de l'influence sur le plasma d'un champ magnétique $\mathbf{B}_{\mathrm{S}}$ axial.

est en désaccord avec celui d'Ogawa [6]. Ogawa obtenait une résonance avec un champ magnétique longitudinal. L'étude de ses expériences permet de remarquer que le champ utilisé est très inhomogène et que ce sont en fait les composantes transversales du champ magnétique $\mathbf{B}_{\mathrm{s}}$ qui provoquent la résonance. Nous avons fait des expériences avec un champ $\mathbf{B}_{\mathrm{S}}$ dont la direction principale est parallèle à l'axe de la décharge mais qui est non uniforme (il possède donc des composantes transversales), on voit alors apparaître la résonance. Pour confirmer cette dépendance entre la résonance et les composantes transversales du champ magnétique $\mathbf{B}_{\mathrm{S}}$, nous avons utilisé un champ magnétique $\mathbf{B}_{\mathrm{S}}$ dont la direction fait un angle $\psi$ avec l'axe de l'enceinte.

La figure 15 montre comment se translate vers les champs $\mathbf{B}_{\mathrm{S}}$ élevés la courbe de résonance lorsque

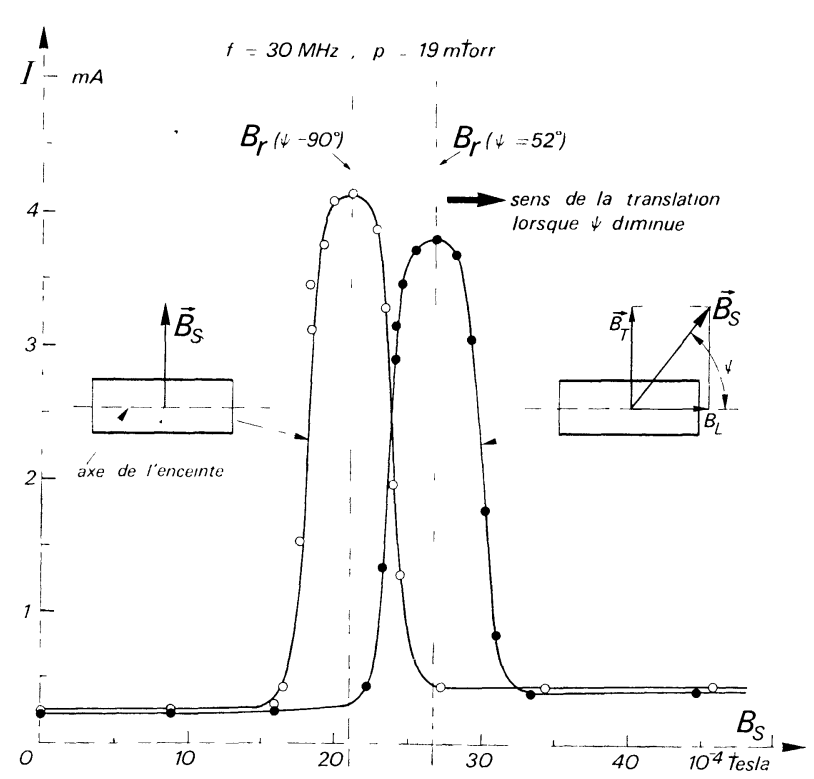

FIG. 15. - Déplacement de la courbe de résonance en fonction de l'inclinaison sur l'axe de la décharge du champ magnétique statique $\mathbf{B}_{\mathbf{s}}$. 
le champ est oblique par rapport à l'axe de l'enceinte à décharge.

Lorsque le champ est purement transversal $\left(\psi=90^{\circ}\right)$ le champ de résonance $B_{\mathrm{r}}$ est compris entre 21 et $22 \times 10^{-4} \mathrm{~T}$. Si le champ magnétique statique fait un angle $\psi=52^{\circ}$ avec l'axe, le champ de résonance a pour valeur $B_{\mathrm{S}}=B_{\mathrm{r}}=27 \times 10^{-4} \mathrm{~T}$, or à cette valeur de $B_{\mathrm{S}}$ correspond une composante transversale $B \mathrm{~T}=B_{\mathrm{S}} \sin \psi \simeq 21 \times 10^{-4} \mathrm{~T}$. Ce qui montre bien que seules les composantes transversales interviennent. Lorsque l'on diminue l'angle $\psi$ la courbe de résonance se déplace vers les champs forts. Si $\psi=0$ il n'y a plus de composante transversale, le champ $\mathbf{B}_{\mathbf{S}}$ est longitudinal, il n'y a pas de résonance.

6. Evolution de la densité électronique $n_{\mathrm{e}}$ et de la température électronique $T_{\mathrm{e}}$ au cours de la résonance. Dans les paragraphes précédents, nous avons vu comment évoluait le courant $I$ dépendant de $n_{\mathrm{e}}$ et de $T_{\mathrm{e}}$ en fonction du champ magnétique statique $B_{\mathrm{S}}$. Il est intéressant d'étudier au moyen de la technique de la sonde double le comportement et la valeur de la densité et de la température électronique du plasma pendant cette résonance. Une étude complète de ces grandeurs a été réalisée [2], nous donnerons seulement ici que quelques résultats importants.

Les figures 16 et 17 montrent les variations de la densité $n_{\mathrm{e}}$ et de la température $T_{\mathrm{e}}$ du plasma en fonction $\mathrm{du}$ champ $\mathbf{B}_{\mathrm{S}}$.

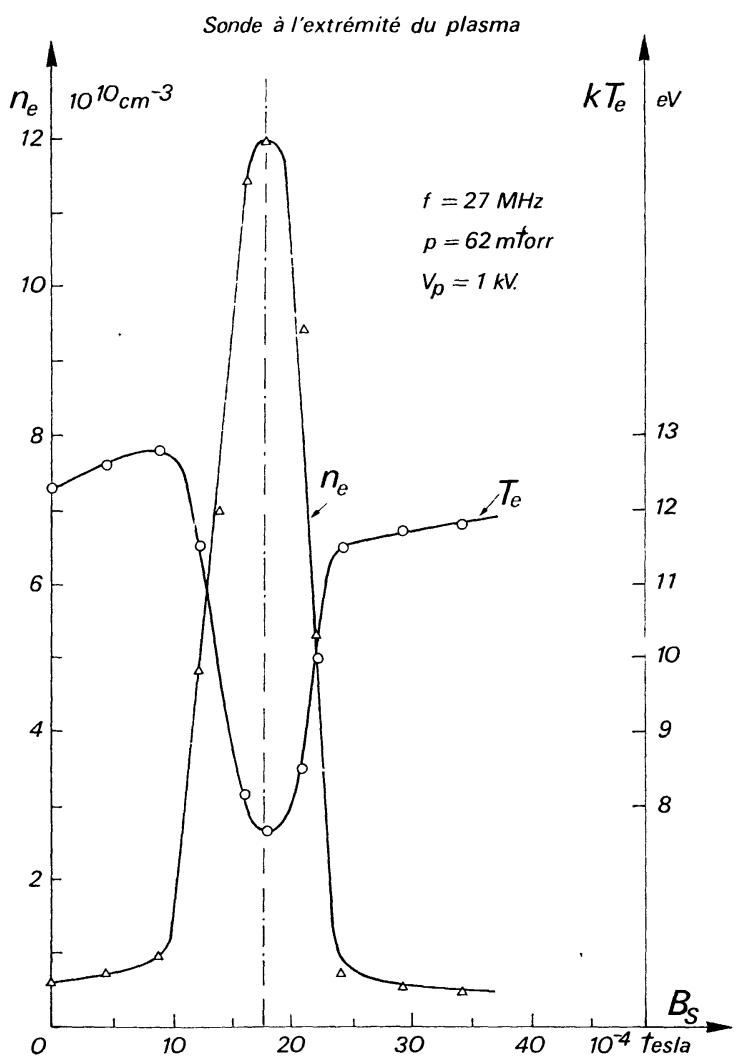

Fig. 16. - Evolution de la densité $n_{\mathrm{e}}$ et de la température $T_{\mathrm{e}}$ pendant la résonance.

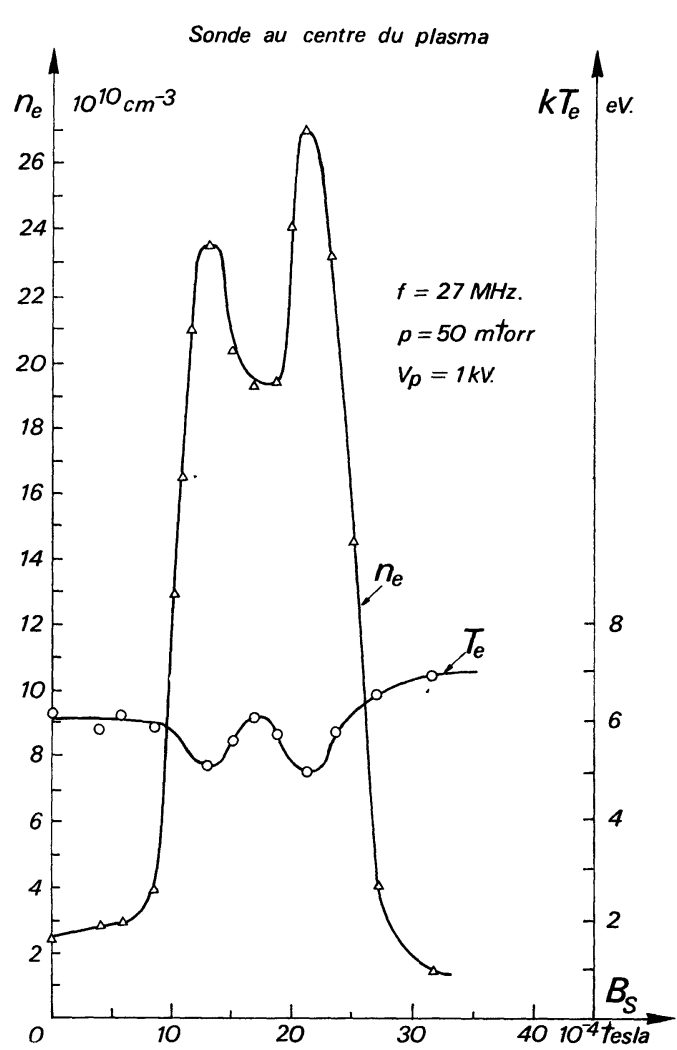

Fig. 17. - Evolution de la densité $n_{\mathrm{e}}$ et de la température $T_{\mathrm{e}}$ pendant la résonance.

On constate que les 'courbes $n_{\mathrm{e}}=g\left(B_{\mathrm{S}}\right)$ sont très semblables aux courbes $I=f\left(B_{\mathrm{S}}\right)$. On retrouve en effet :

- Le maximum de densité pour $B_{\mathrm{S}}=B_{\mathrm{r}}$ tel que $f_{\mathrm{c}} \simeq 2 f$.

- Le dédoublement du pic de résonance dans les mêmes intervalles de pressions et de puissance $\mathrm{HF}$ que pour l'intensité $I$.

Nous constatons que la résonance permet d'augmenter de manière considérable la densité du plasma (le taux d'augmentation est supérieur à 10). La puissance fournie au plasma augmente beaucoup à la résonance, le résultat précédent montre ainsi que cette puissance et la densité électronique $n_{\mathrm{e}}$ sont intimement liées. Il est cependant difficile de déterminer lequel de ces deux facteurs régit l'autre lors de la résonance.

Il est important de remarquer que seul le phénomène de résonance permet d'augmenter la densité électronique dans un rapport aussi élevé. Il existe tout un domaine de pressions situé avant 20 mtorr pour lequel, en l'absence de champ magnétique transversal $\mathbf{B}_{\mathrm{s}}$, il est impossible d'obtenir avec la puissance HF dont nous disposons une décharge induite, c'est-à-dire en fait un plasma avec une densité $n_{\mathrm{e}}$ de l'ordre de $10^{11} \mathrm{~cm}^{-3}$. Par contre entre 10 et 20 mtorr le champ magnétique statique $\mathbf{B}_{\mathbf{S}}$ permet d'obtenir la résonance, c'est-à-dire le transfert d'énergie entre 
l'oscillateur et le plasma et l'accroissement de la densité $n_{\mathrm{e}}$.

L'évolution de la température électronique $T_{\mathrm{e}}$ est toujours de sens opposé au sens de variation de la densité $n_{\mathrm{e}}$. Ainsi les figures précédentes montrent qu'à la résonance la température électronique diminue, cette diminution est plus importante à l'extrémité du plasma (de 13 à $6 \mathrm{eV}$ ) qu'au centre du plasma (de 9 à $6 \mathrm{eV}$ ).

A la résonance la variation de densité $n_{\mathrm{e}}$ sur l'axe de la décharge, de l'extrémité au centre du plasma, est de l'ordre de 1 à $3\left(10^{11}\right.$ à $\left.2,7 \times 10^{11} \mathrm{~cm}^{-3}\right)$, la variation de la température étant de l'ordre de $3 \mathrm{eV}$. Ces valeurs sont à rapprocher de celles obtenues en dehors de la résonance, le rapport des densités varie entre 5 et 10 , la différence de température étant de l'ordre de $6 \mathrm{eV}$. Ceci semble indiquer que lors de la résonance le plasma tend à devenir plus homogène, peut-être à cause de la diffusion longitudinale des particules dans le champ magnétique statique $\mathbf{B}_{\mathrm{S}}$.

7. Conclusion. - 7.1 RÉSultats obtenus. Cette étude nous a permis de déterminer et de préciser les différents paramètres expérimentaux qui interviennent dans le phénomène de résonance du plasma HF. Nous avons ainsi mieux défini ce phénomène. En particulier nous avons montré :

a) Que la résonance apparaît lorsque la fréquence gyromagnétique des électrons est voisine du double de la fréquence d'excitation de la décharge.

b) Que la résonance n'existe qu'à l'intérieur d'un intervalle de pressions.

La borne inférieure de ce domaine (10 mtorr) paraît fixe en fonction de la fréquence et de la puissance d'excitation.

La borne supérieure par contre, semble liée à la puissance d'excitation de la décharge, cette limite étant d'autant plus élevée que la puissance HF est grande, c'est-à-dire que le champ électrique induit est plus élevé. Avec la plus forte puissance HF disponible dans nos expériences, la limite supérieure du domaine d'existence se situe vers 70 mtorr.

c) L'importance de la direction du champ magnétique statique $\mathbf{B}_{\mathrm{s}}$ par rapport à la direction du champ électrique d'excitation.

Nous avons montré que seules les composantes transversales (perpendiculaires à l'axe du système) intervenaient pour fixer la valeur du champ de résonance. Si ces composantes n'existent pas, il n'y a pas de résonance.

d) Que le mode de couplage du générateur HF au plasma est déterminant. A ce mode de couplage est lié l'existence du champ électrique induit.

Il semble ainsi que la présence du champ induit $\mathbf{E}_{\boldsymbol{\theta}}$ soit indispensable à l'observation de la résonance.

D'autre part, la largeur de la courbe de résonance semble liée à l'amplitude du champ électrique longitudinal.
Nous avons également mis en évidence l'influence de la puissance du générateur haute fréquence sur la forme de la courbe de résonance. Pour des puissances suffisamment élevées, le pic de résonance se dédouble en deux pics symétriquement placés par rapport au pic initial.

L'étude de l'évolution de la densité électronique du plasma, en fonction du champ magnétique statique, nous a montré que cette densité augmentait beaucoup à la résonance. Cet accroissement est étroitement lié au fait qu'à la résonance l'impédance du plasma et celle du générateur semblent s'adapter, permettant ainsi un transfert de puissance optimum.

La résonance permet d'obtenir aisément des densités électroniques de l'ordre de $5 \times 10^{11} \mathrm{~cm}^{-3}$. La densité électronique peut être modifiée à volonté en agissant sur le champ magnétique statique. La résonance apparaît donc comme un moyen particulièrement adapté pour accroître et contrôler la densité d'une décharge en haute fréquence dans l'hydrogène.

7.2 Comparaison AVEC LES aUtres travaux. Pratiquement, la seule possibilité de comparaison porte sur le point $\dot{a}$ ). Dans nos expériences, le champ de résonance correspond à une fréquence gyromagnétique $f_{\mathrm{c}}$ toujours voisine, par valeurs inférieures, de deux fois la fréquence d'excitation, soit $f_{\mathrm{c}} / f \simeq 2$, et ceci pour des densités électroniques $n_{\mathrm{e}}$ à la résonance qui varient entre $5 \times 10^{9}$ et $5 \times 10^{11} \mathrm{~cm}^{-3}$.

Pour O. Sager [11], dans le cas d'une décharge dans une enceinte cylindrique de diamètre $6,2 \mathrm{~cm}$, contenant de l'argon à la pression de $5 \times 10^{-3}$ torr, le rapport $f_{\mathrm{c}} / f$, à la résonance, évolue entre 2,1 et 3,1 , la densité $n_{\mathrm{e}}$ à la résonance évoluant entre $4 \times 10^{10}$ et $3,5 \times 10^{11} \mathrm{~cm}^{-3}$. Si le diamètre de l'enceinte atteint $8,3 \mathrm{~cm}, f_{\mathrm{c}} / f$ évolue entre 2,9 et 5 , pour le même intervalle de densité. Ainsi, il apparaît que la densité à la résonance est une fonction pratiquement linéaire du champ magnétique de résonance $B_{\mathrm{r}}$. Par contre, dans notre cas, la densité à la résonance apparaît toujours pour la même valeur du champ magnétique statique.

Cette dépendance entre $n_{\mathrm{e}}$ et $B_{\mathrm{S}}$ a été analysée théoriquement par B. Pfieffer et O. Sager. Pour cela, ils étudient la propagation du champ électromagnétique à l'intérieur du plasma. De nombreuses simplifications sont introduites dans ce calcul et en définitive le problème consiste à étudier la propagation du champ électromagnétique à l'intérieur d'une lame de plasma. L'adaptation de cette théorie au cas d'un plasma cylindrique a été réalisée par Szuszczewicz, ce calcul conduit à l'expression suivante de la densité $n_{\mathrm{e}}$ à la résonance :

$$
\begin{aligned}
\left(n_{\mathrm{e}}\right)_{\mathrm{res}} & =\left(\frac{c^{2} \pi^{2} \varepsilon_{0} m}{e^{2}}\right) \times \\
& \times \frac{\pi^{2} p^{2}\left(\lambda_{\mathrm{i}}\right)\left(\beta_{\mathrm{res}}-\sqrt{2}\right)^{3}}{\left(4 d^{2} / \sqrt{2}\right)\left(\beta_{\mathrm{res}}-\sqrt{2}\right)^{2}+\pi^{4} k T_{\mathrm{e}} p^{2}\left(\lambda_{\mathrm{i}}\right) / m \omega^{2}}
\end{aligned}
$$


avec :

$d$ diamètre de l'enceinte à décharge ;

$\beta_{\text {res }}=\left(f_{\mathrm{c}} / f\right)$ à la résonance ;

$p\left(\lambda_{\mathbf{i}}\right)$ coefficient numérique faisant intervenir le libre parcours moyen des ions par rapport aux dimensions de l'enceinte à décharge.

Pour comparer cette théorie à nos résultats expérimentaux, nous avons tracé la courbe donnant $\left(n_{\mathrm{e}}\right)_{\text {res }}$ en fonction de $f_{\mathrm{c}} / f$, d'après la formule précédente et en choisissant les valeurs de $d$ et de $T_{\mathrm{e}}$ en rapport avec nos conditions expérimentales, à savoir :

$$
d=40 \mathrm{~mm}, \quad k T_{\mathrm{e}} \simeq 8 \mathrm{eV} .
$$

La courbe obtenue numériquement est représentée sur la figure 18. Nous avons également reporté sur cette figure la zone enveloppe de nos résultats expérimentaux (en hachures), la densité à la résonance évolue dans cette zone.

On constate sur ce graphique la grande influence du diamètre $d$ de l'enceinte à décharge, alors que nos expériences montrent que pour des diamètres plus petits, compris entre 20 et $60 \mathrm{~mm}$, le phénomène est inchangé.

Cette théorie ne fait pas intervenir de façon nette la pression du gaz et elle ne respecte pas la distribution du champ électromagnétique dans une bobine hélicoïdale.

Nous pensons que la théorie proposée dans les références [9]-[11]-[12], n'explique pas nos résultats

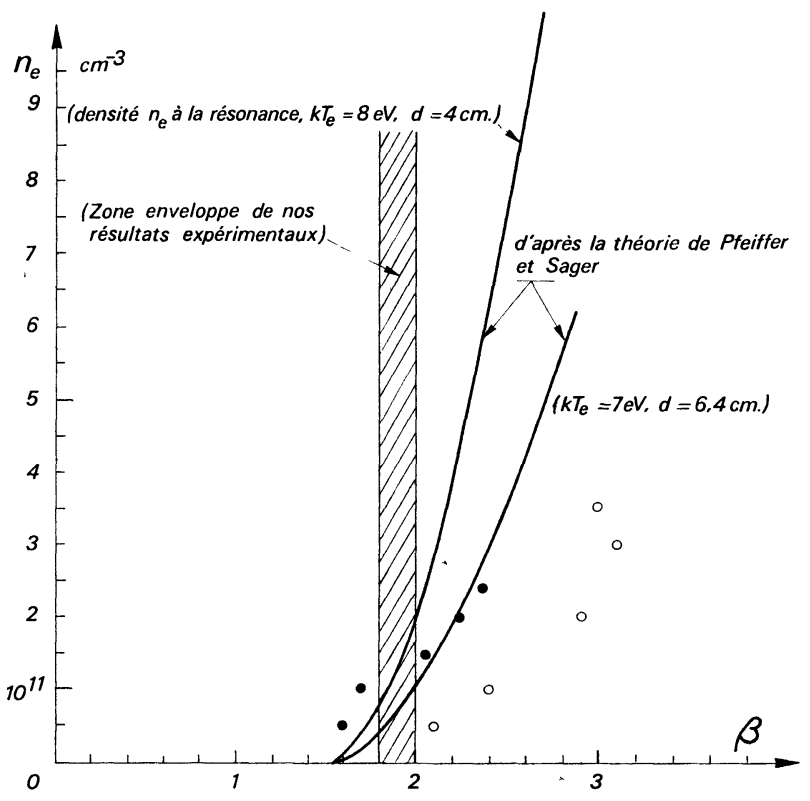

FIG. 18. - Comparaison des prévisions théoriques avec nos résultats expérimentaux. $\bigcirc$ Résultats expérimentaux de Sager [11] dans l'argon ( $p=5 \times 10^{-3}$ torr, $f=27 \mathrm{MHz}, d=6,2 \mathrm{~cm}$ ).

- Résultats de Pfeiffer [8] $(d=6,4 \mathrm{~cm})$.

expérimentaux et que vraisemblablement les phénomènes observés sont différents. Ces différences peuvent en partie être dues à la non-identité dans la définition de la résonance, dans la configuration du système et dans la méthode expérimentale.

\section{Bibliographie}

[1] Collet, L. H., Grolleau, B., J. Physique 29 (1968) C 3-209.

[2] Grolleau, B., Thèse Sc. Phys., Nantes (1973) n ${ }^{\circ}$ CNRS AO 8300.

[3] Neuert, H. Z., Z. Naturforsch. a. 4-a (1949) 449.

[4] Swingle, J. S., Swann, C. B., Rev. sci. Instrum. 23 (1952) 636.

[5] Gabovich, M. D., Zh. Tekh. Fiz. SSSR 28 (1958) 872

[6] Ogawa, I., Abe, N., Nucl. Instrum. Meth. 16 (1962) 227.

[7] Williams, J. F., Rev. Sci. Instrum. 37 (1966) 1205.

[8] Pfeiffer, B., J. Appl. Phys. 37 (1966) 1624.

[9] Pfeiffer, B., J. Appl. Phys. 37 (1966) 1628.

[10] Oechsner, H., Gas Discharge IEE Conf., 187, Londres (1970).

[11] SAGER, O., Thèse Institut des Hautes Fréquences, Zurich (1970).

[12] Szuszczewicz, E. P., J. Appl. Phys. 42 (1971) 4794.
[13] Allen, J. E., Segre, S. E., Il Nuovo Cimento XXI (1961) 980.

[14] Anicin, B. A., Marsicanin, B. S., Proc. 6e ICIPG Paris 2 (1963) 491.

[15] Johnson, E. O., Malter, L., Phys. Rev. 80 (1950) 58.

[16] Swift, J. D., Schwar, M. J. R., Electrical Probes for Plasma Diagnostics (Iliffe Books, London) 1970.

[17] Contaxes, N., Hatch, A. J., J. Appl. Phys. 40 (1969) 3548.

[18] KeEFER, D. R., Thèse Université de Floride, Gainesville (1967).

[19] Turban, G., Thèse 3e Cycle, Nantes (1971).

[20] Allis, W. P., Brown, S. C., Phys. Rev. 87 (1952) 419.

[21] Collet, L. H., Grolleau, B., C. R. Hebd. Séan. Acad. Sci. B 262 (1966) 1668.

[22] Dodge, N. B., Kristiansen, M., Dougal, A. A., Rev. Sci. Instrum. 37 (1966) 1455. 